\title{
Comparison of Ultrafast Papanicolaou stain with Standard Papanicolaou stain for cervical smear
}

\author{
Sujata Pudasaini ${ }^{1}$, Rakesh Pathak ${ }^{1}$, Kricha Pande ${ }^{1}$, Shraddha Koirala ${ }^{1}$ \\ ${ }^{\prime}$ Department of Pathology, Nepal Medical College Teaching Hospital, Kathmandu, Nepal.
}

\section{Keywords: \\ Cervix; \\ Pap smear; \\ Papanicolaou; \\ Staining; \\ Ultrafast;}

\begin{abstract}
Background: After the first evolution of Papanicolaou smear, it has been successfully used to screen cervical cancer. With modifications in the staining method of Pap stain, the lesser staining time and unequivocal cell morphology has been achieved by Ultrafast Papanicolaou (UFP) stain. The aim of this study is to compare the UFP stain and Standard Papanicolaou (SP) stain in cervical pap smears on the basis of background, cell morphology, nuclear details and overall staining.
\end{abstract}

Materials and methods: This is a prospective hospital based study conducted in a tertiary hospital, Nepal Medical College over a period of six months (January 2017 to June 2017). Cervical pap smears received in the department of Pathology is subjected to Standard papanicolaou (SP) stain and Ultrafast papanicolaou (UFP) stain for comparison.

Results: Total of 368 cervical pap smears were subjected to SP stain and UFP stain. Mean age of the patient was $36.57+-10.06$ years. The quality of UFP stain was assessed by considering the background, cell morphology, nuclear details and overall staining. Difference in regards to the interpretation and background was not significant when SP stain was compared to UFP stain. However, cell morphology, nuclear details and overall staining were far better in smears stained with UFP stain.

Conclusion: Pap smear is the simple and effective screening tool for cervical lesions. With modifications in staining method and introduction of UFP stain, the result is lesser staining time and excellent morphological quality. Hence, UFP stain has become the choice of stain and can be used on a regular basis.

\section{Correspondence:}

Dr. Sujata Pudasaini, MD

Associate Professor, Department of Pathology,

Nepal Medical College Teaching Hospital, Kathmandu, Nepal

ORCID ID: 0000-0001-9699-8204

Email: sujatapudasaini@gmail.com

Received : July $1^{\text {st }} 2018$; Accepted : August 15 $5^{\text {th }} 2018$; Published : September $1^{\text {st }} 2018$

Citation: Pudasaini S, Pathak R, Pande K, Koirala S. Comparison of Ultrafast Papanicolaou stain with Standard Papanicolaou stain for cervical smear. J Pathol Nep 2018;8:1378-83. DOI: 10.3126/ jpn.v\%vi\%i.20890

Copyright: This is an open-access article distributed under the terms of the Creative Commons Attribution 4.0 International License, which permits unrestricted use, distribution, and reproduction in any medium, provided the original author and source are credited.

\section{INTRODUCTION}

Cervical Pap smear is a simple, convenient, cost effective and reliable test for easy screening of cervical lesions. Since its introduction, there has been a dramatic reduction in the incidence and mortality of invasive cervical cancer world wide. ${ }^{1-3}$ Pap stain is the preferred stain for gynaecological and non gynaecological cytology smear., ${ }^{4,5}$ Pap stain yields polychromatic transparent staining reaction with crisp nuclear and cytoplasmic features. It was first developed by Dr. George Nicholas Papanicolaou in 1942 to know the variation in cellular maturity and metabolic activity in vaginal smears. ${ }^{4}$ Pap stain clearly distinguish between 
Table 1: Procedure - Ultrafast pap (UFP) stain and Standard pap (SP) stain :

\begin{tabular}{|c|c|}
\hline Ultrafast pap (UFP) stain & Standard pap (SP) stain \\
\hline 1. Slides received in $95 \%$ ethanol & 1. Slides received in $95 \%$ ethanol is removed \\
\hline $\begin{array}{l}\text { 2. Hydration }-10 \text { passes under the running tap water. Blot off excess } \\
\text { water. }\end{array}$ & 2. $70 \%$ ethanol - 15 dips \\
\hline 3. Dip in nuclear stain for 45 seconds. Wash under tap water & 3. Wash in running tap water for 30 seconds \\
\hline $\begin{array}{l}\text { 4. Add } 3 \text { drops of Scott's tap water buffer. Wash in running tap water } \\
\text { after } 10 \text { seconds. Blot off excess water }\end{array}$ & $\begin{array}{l}\text { 4. Stain with Harris's Haematoxylin for } 2 \text { minutes. Wash under tap water } \\
\text { for } 30 \text { seconds. }\end{array}$ \\
\hline 5. Dip in Dehydrant for 30 seconds - two changes & $\begin{array}{l}\text { 5. Rinse in acid alcohol } 1 \%-2 \text { dips. Wash in running tap water for } 3 \\
\text { minutes }\end{array}$ \\
\hline $\begin{array}{l}\text { 6. Dip in working cyto stain (prepared by mixing (cytostain A and } \\
\text { cytostain B) } 15 \text { seconds. Wash and blot off the excess water }\end{array}$ & 6. Dip in $70 \%$ ethanol -15 dips \\
\hline 7. Dip in dehydrant for 30 seconds then dry & 7. Dip in $90 \%$ ethanol - 15 dips \\
\hline 8. Dip in xylene- two changes & 8. Stain with OG 6 for 2 minutes \\
\hline \multirow[t]{5}{*}{ 9. Mount with DPX } & 9. Rinse with $95 \%$ ethanol -2 changes, 15 dips in each \\
\hline & 10. Stain with EA-50 for 5 minutes. \\
\hline & 11. Rinse in $95 \%$ ethanol -2 changes, 15 dips each \\
\hline & 12. Xylene -2 changes -2 minutes each \\
\hline & 13. Mount with DPX \\
\hline Total time taken: 2.5 minutes & Total time taken: 20 minutes \\
\hline
\end{tabular}

basophilic and acidophilic cell components and gives the detailed chromatin pattern making the nuclear details very good. The pap stain has 3 solutions having 6 dyes. Solution 1 has hematoxylin stain, a basic nuclear stain. Solution 2 has OG-6 with phosphotungstic acid an acidic cytoplasmic stain where orange $\mathrm{G}$ stains keratin with bright intense orange and phosphotungstic acid intensify the color. Solution 3 has EA, a polychromatic stain having 3 components for staining cytoplasm in different cells.

SP stain is time consuming and takes 20- 30 minutes. ${ }^{4}$ As practiced conventionally SP stain use a substantial quantity of alcohol which hinders its use as a mass screening tool in low resource settings. It takes a long time to complete the staining. ${ }^{6}$ Since its evolution, the pap stain has undergone various modifications. ${ }^{5-7}$

With the need for minimal turn around time for assessing the cervical pap smears has encouraged innovations in staining procedures that require lesser staining time with unequivocal cell morphology. Modifications have been developed in pap stain to improve the staining quality and to minimize staining time. Ultrasfast papanicolaou (UFP) stain was introduced by Yang and Alvarez in 1995. 5,7,8 UFP stain is a hybrid of the technique by Romanowsky and conventional pap stain to reduce the staining times to less than 2 minutes. ${ }^{7,9}$

\section{MATERIALS AND METHODS}

This is a prospective hospital based study conducted in a tertiary hospital, Nepal Medical College over a period of six months (January 2017 to June 2017). Permission to carry out the study was obtained from Institutional Review Committee of the institute. Cervical pap smears (two slides for each case) received in the department of Pathology in the study period was included. Out of the two slides in each case, one was stained with SP stain and another was stained with UFP stain. Table 1 shows the staining procedure of UFP stain and SP stain.

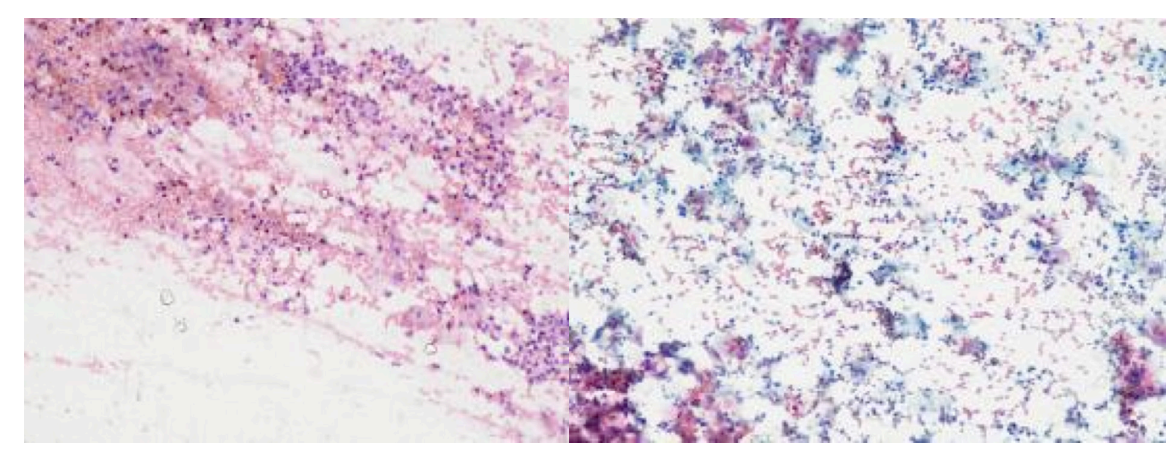

Figure 1: A. Standard pap stain showing hemorrhage in the background (low power; $x$ 100). B. Ultrafast pap stain showing hemorrhage in the background with better staining quality (low power; $x$ 100) 
Table 2: Smears with hemorrhagic background (b/k) in UFP stain and SP stain

\begin{tabular}{lcccr}
\hline & \multicolumn{2}{c}{ Ultrafast pap (UFP) stain } & \multicolumn{2}{c}{ Standard pap (SP) stain } \\
\cline { 2 - 5 } Background & \multicolumn{1}{l}{ Number } & Percentage (\%) & Number & Percentage (\%) \\
\cline { 2 - 5 } & 65 & 17.7 & 63 & 17.1 \\
\hline Hemorrhage & 303 & 82.3 & 305 & 82.9 \\
No Hemorrhage & $\mathbf{3 6 8}$ & $\mathbf{1 0 0}$ & $\mathbf{3 6 8}$ & $\mathbf{1 0 0}$ \\
\hline Total & & & \\
\hline
\end{tabular}

Table 3: Comparison of inflammatory background in UFP stain and SP stain

\begin{tabular}{|c|c|c|c|c|}
\hline \multirow{2}{*}{ Inflammation } & \multicolumn{2}{|c|}{ Ultrafast pap (UFP) stain } & \multicolumn{2}{|c|}{ Standard pap (SP) stain } \\
\hline & Number & Percentage (\%) & Number & Percentage (\%) \\
\hline Dense & 193 & 52.5 & 210 & 57.1 \\
\hline Moderate & 113 & 30.7 & 110 & 29.9 \\
\hline Minimum & 48 & 13 & 42 & 11.4 \\
\hline Clean & 14 & 3.8 & 6 & 1.6 \\
\hline Total & 368 & 100 & 368 & 100 \\
\hline
\end{tabular}

Table 4: Preservation of cell morphology of UFP stain and SP stain

\begin{tabular}{lcccc}
\hline \multirow{2}{*}{ Cell morphology } & \multicolumn{2}{c}{ Ultrafast pap (UFP) stain } & Standard pap (SP) stain \\
\cline { 2 - 5 } & & & & Number \\
\hline Well preserved & Number & Percentage (\%) & 51 & 13.9 \\
\hline Moderately Preserved & 287 & 78 & 307 & 83.4 \\
\hline Not preserved & 79 & 21.5 & 10 & 2.7 \\
\hline Total & 2 & 0.5 & $\mathbf{3 6 8}$ & $\mathbf{1 0 0}$ \\
\hline
\end{tabular}

The reporting was done using The Bethesda System 2014 of reporting cervical smears. The four parametersbackground, cell morphology, nuclear details and overall staining were considered. These findings and interpretation were noted separately for UFP stain and SP stain. Data were then entered in Microsoft office excel 2003 then analyzed using Statistical Package for the Social Sciences- 16 (SPSS 16).

\section{RESULTS}

A Total of 368 cervical pap smears were analyzed during the study period. The minimum age of the patient was 19 years and maximum was 75 years. The mean age was 36.57 +/10.06. The time taken for staining the smears with UFP stain and SP stain was 2.5 minutes and 20 minutes respectively. The quality of these two stains was assessed based on the background, preservation of cell morphology, nuclear details and overall staining. The quality of UFP stain was better when compared to SP stain.
Out of the total cases, there was not much of variation in background of smears in case of UFP stain and SP stain when the smear was hemorrhagic and inflammatory (Table 2 and Table 3 ).

Hemorrhagic background was seen in $17.7 \%$ and $17.1 \%$ in UFP stain and SP stain respectively. RBCs were standing out in the background and were not obscuring the epithelial cells in the smear stained by UFP stain (fig.1A\&B) Even in the presence of dense inflammation, the cell differentiation was good in UFP stain (fig.2A\&B). The preservation of cell morphology, nuclear details and overall staining was much better in UFP stain (Table 4, 5 and 6).

There was no difference in the interpretation / result of cervical smears of UFP stain and SP stain. However, there is a little variation in the non neoplastic findings like reactive cellular changes and in the identification of organisms. (Table 7) 
Table 6: Overall staining of UFP stain and SP stain

\begin{tabular}{|c|c|c|c|c|}
\hline \multirow{2}{*}{ Overall staining } & \multicolumn{2}{|c|}{ Ultrafast pap (UFP) stain } & \multicolumn{2}{|c|}{ Standard pap (SP) stain } \\
\hline & Number & Percentage (\%) & Number & Percentage (\%) \\
\hline Good & 295 & 80.2 & 36 & 9.8 \\
\hline Moderately good & 71 & 19.3 & 305 & 82.9 \\
\hline Bad & 2 & 0.5 & 27 & 7.3 \\
\hline Total & 368 & 100 & 368 & 100 \\
\hline
\end{tabular}

\section{DISCUSSION}

The pap smear has been used for cervical cancer screening for more than 50 years. The conventional SP stain is complex with multiple steps of greatly varying times making it liable to laboratory errors. ${ }^{6}$ Since the introduction of pap stain, it has undergone various modifications. ${ }^{4}$ This modification in the procedure of pap staining has come up with excellent result in the form of better staining quality and time conservation. ${ }^{8}$

Our study had shown that the time taken to stain the cervical smear was 2.5 minutes and 20 minutes respectively in UFP stain and SP stain. Gupta et al showed similar finding in his study where the time taken to stain the same number of pap smear slides was reduced considerably to 3 minutes compared to SP stain in which the time taken was 20 minutes. ${ }^{10}$ The same finding was seen in a study done by
Kamal et al. ${ }^{9}$

Almost the same numbers of cervical smears stained with UFP stain and SP stain show haemorrhage in the background. However, the morphology of RBCs and overall background was much clearer in UFP stained smears. In contrast to our study, Choudhary et al and Shinde et al had shown that UFP stained smears had clear RBC free background in their studies. ${ }^{8,11}$ Further, Arul et al showed that UFP stain provides a clean background devoid of RBCs than conventional SP stain when the smears were rehydrated by normal saline. ${ }^{12}$

When the background inflammations was compared in two smears stained by UFP stain and SP stain, it was almost the similar finding which suggested that it does not differentiate between the density of inflammation but morphology was clearer in UFP. When the morphology is clear there is a less chance of missing and help in making the right diagnosis. ${ }^{7}$

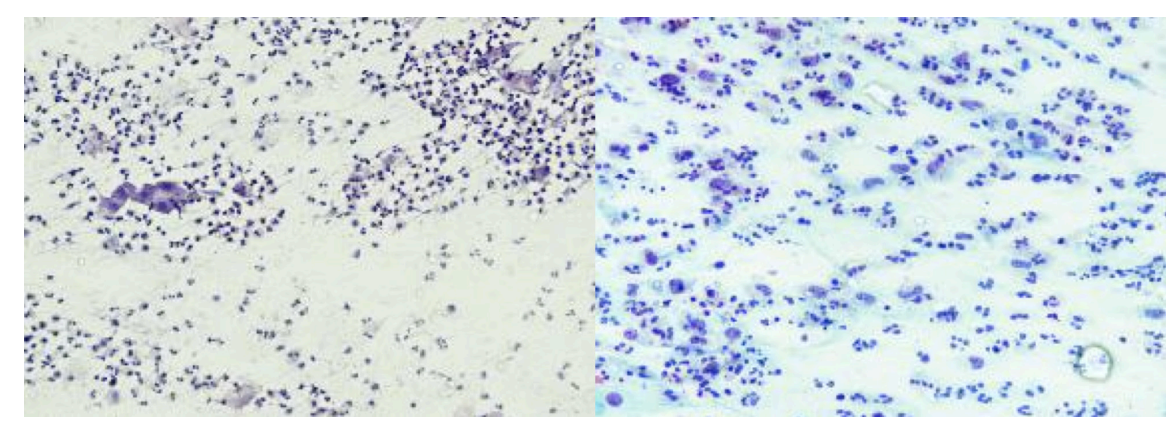

Figure 2: A. Standard pap stain showing histiocytes and neutrophils in the background in inflammatory smear (low power; $x$ 100). B. Ultrafast pap stain showing histiocytes and neutrophils in the background in inflammatory smear (low power; $\mathrm{X100)}$

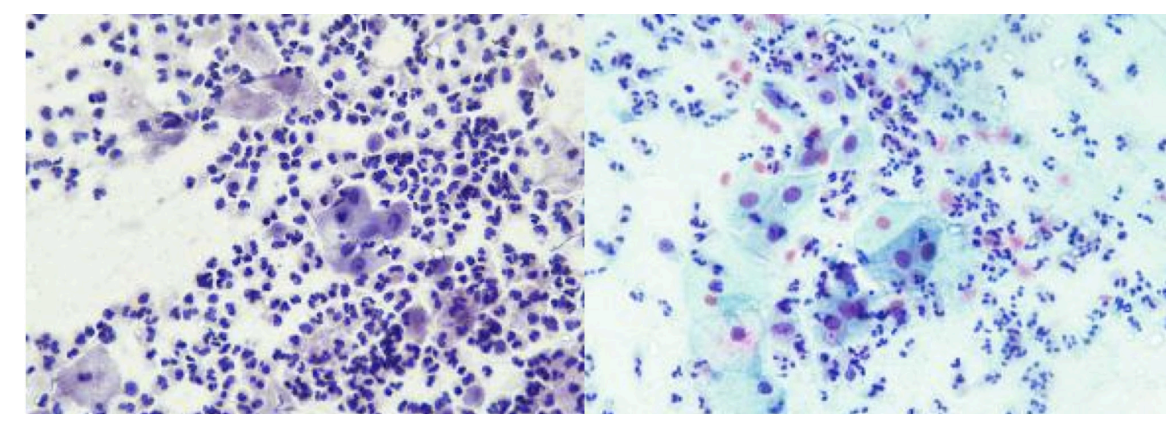

Figure 3: A. Standard pap stain- reactive cellular changes (x 200). B. Ultrafast pap stain- reactive cellular (X200) 
Table 7: Interpretation/ Result of cervical smear in UFP stain and SP stain

\begin{tabular}{|c|c|c|c|c|}
\hline \multirow{2}{*}{ Interpretation/ Result } & \multicolumn{2}{|c|}{ Ultrafast pap (UFP) stain } & \multicolumn{2}{|c|}{ Standard pap (SP) stain } \\
\hline & Number & Percentage (\%) & Number & Percentage $(\%)$ \\
\hline Negative for intraepithelial lesion (IEL) or malignancy & 311 & 84.5 & 283 & 76.9 \\
\hline Obscuring Inflammation & 5 & 1.4 & 8 & 2.2 \\
\hline Reactive cellular changes (fig.3A\&B) & 21 & 5.7 & 40 & 10.9 \\
\hline Shift in flora suggestive of Bacterial vaginosis & 18 & 4.9 & 25 & 6.8 \\
\hline Trichomonas vaginalis (fig.4A\&B) & 10 & 2.7 & 9 & 2.4 \\
\hline LSIL (Low grade squamous IEL) & 3 & 0.8 & 3 & 0.8 \\
\hline Total & 368 & 100 & 368 & 100 \\
\hline
\end{tabular}

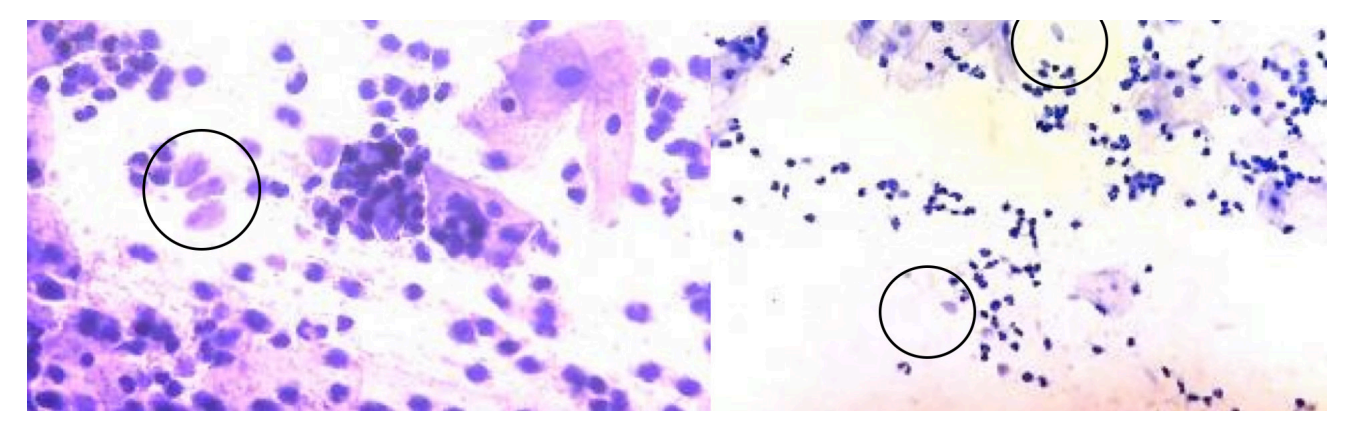

Figure 4A: Standard pap stain- Trichomonas vaginalis (X200). B. Ultrafast pap stain- Trichomonas vaginalis (X100)

Nuclear details, background, cell morphology and overall staining are essential features for successful screening. ${ }^{\text {? }}$

In our study, the cell morphology was well preserved in UFP stained smears. The staining quality of nuclear chromatin and overall staining was much better in UFP stained smears than SP stained smears. This correlates very well with other studies. ${ }^{5-8,13,14}$

Our study showed that there was no difference in the interpretation/ result of cervical pap smears stained by two methods. This correlates well with study done by Gachi et al. ${ }^{6}$ However, there were minor variations in the non neoplastic findings. Reactive cellular changes were more commonly seen in SP stained smears compared to UFP stained smears. This could be due to the better nuclear details achieved in UFP stained smears which minimized the misinterpretation of reactive cellular changes in many cervical smears.

Similarly, the number of cases with shift in flora was more in SP stained smears. With more preserved cell morphology and better overall staining quality there is reduction in the number of cases of shift in flora in UFP stained smears. Diagnosis of bacterial vaginosis is important as it is associated with serious health problems. Cervical pap smears had been beneficial in diagnosing the infections apart from cancer screening. ${ }^{15,16}$ When it comes to Trichomonas vaginalis, the effectiveness of diagnosis is not much higher on pap smears but it has been proved to be beneficial in detecting these infections. ${ }^{16}$ In our study one case of trichomonas vaginalis was missed in SP stained smear and was identified in UFP stained smear. The morphology of trichomonas vaginalis was also better in UFP stained smears.

Lesser staining time and an excellent morphological quality is the need for any cytopathology set up. UFP stain easily fulfils these criteria than conventional SP stain. ${ }^{13,14}$ The simplicity of the procedure in UFP stain reduced the risk of errors while staining as the procedure is short and simple. ${ }^{5}$

\section{CONCLUSION}

Pap staining procedures have undergone a lot of changes since its evolution. The UFP stain is a simple and technician friendly procedure that does not compromise on staining quality and diagnostic standards. It can be used as a suitable time consuming pap staining procedure.

\section{ACKNOWLEDGEMENT}

We would like to thank Mr. Prem Prasad Pant from department of Community Medicine of Nepal Medical College for his help in data analysis.

\section{Conflict Interest: None}




\section{REFERENCES}

1. Hirachand S, Bajracharya J, Pradhanang S, Lama S. Detection of abnormal cervical cytology in papanicolaou smears in a tertiary care center. J Nepal Med Asssoc 2013;52: 462-5

2. Ranabhat SK, Shrestha R, Tiwari M. Analysis of abnormal epithelial lesions in cervical pap smears in Mid Western Nepal. J Pathol Nepal 2011;1:30-3. Crossref

3. Bukhari MH, Saba K, Qamar S, Majeed MM, Niazi S, Naeem S. Clinicopathological importance of papanicolaou smears for the diagnosis of premalignant and malignant lesions of the cervix. $\mathrm{J}$ Cytol 2012;29:20-5. $\underline{\text { Crossref }}$

4. Kalyani R. Evolution of pap stain. Biomed Res Ther 2016; 3: 490500

5. Patrikar A, Joshi A, Govardhan V, Dongre T. Comparison of modified ultrafast papanicolaou stain with the standard papanicolaou stain in cytology of various organs. Intl J Sci Res Pub 2016;6:354-9

6. Gachie RN, Muchiri LW, Ndungu JR. A comparison of modified and standard papanicolaou staining methods in the assessment of cervical smears at Kenyatta National Hospital. East Afr Med J 2011;88:24450

7. Khajuria R, Sharma M, Singh K, Koul KK. A comparative study to see the utility of modified ultrafast papanicolaou (MUFP) stain over standard pap stain in routine FNA smears. J Evid Based Med Health 2016;3:2762-5. Crossref

8. Choudhary P, Sudhamani S, Pandit A, Kiri VM. Comparison of modified ultrafast papanicolaou stain with the standard rapid papanicolaou stain in cytology of various organs. J Cytol 2012;29:241-5. Crossref
9. Kamal MM, Kulkarni MM, Wahane RN. Ultrafast papanicolaou stain modified for developing countries: efficacy and pitfalls. Acta cytological 2011;55:205-12. $\underline{\text { Crossref }}$

10. Gupta S, Chachra KL, Bhadola P, Sodhani P. Modified papanicolaou staining protocol with minimum alcohol use: a cost cutting measure for resource-limited settings. Cytopathology, 2010;21:229-33. $\underline{\text { Crossref }}$

11. Shinde PB, Pandit AA. Application of modified ultrafast papanicolaou stain in cytology of various organs. Diagn Cytopathol 2006;34:1359. $\underline{\text { Crossref }}$

12. Arul P, Eniya S, Pushparaj M, Masilamani M, Kanmani P, Lingasamy C. Comparative assessment of conventional papanicolaou and modified ultrafast papanicolaou stains in fine needle aspiration samples and body fluids. J Cytol 2018;35:46-50. $\underline{\text { Crossref }}$

13. Thakur M, Guttikonda VR. Modified ultrfast papanicolaou staining technique: a comparative study. J Cytol 2017;34:149-53.

14. Alwahaibi NY, Alsubhi MS, Aldairi N, Alshukaili A, Bai UR. Comparison of ultrafast papanicolaou stain with standard papanicolaou stain in body fluids and fine needle aspiration specimens. J Lab Phy 2016;8:19-24.

15. Vandana G, Kumar KR, Khan S, Anil S. Cytological findings of bacterial vaginosis in routine pap smears - a two years institutional study. J Dent Med Sci 2018;17:68-78

16. Avwioro OG. Diagnosis of trichomonas in pap smears; how effective is it? Eur J Exp Biol 2011;1:10-3. 